\title{
From form factors to generalized parton distributions
}

\section{Markus DIEHL*}

Deutsches Elektronen-Synchroton DESY, 22603 Hamburg, Germany

E-mail: markus.diehledesy.de

I present an extraction of generalized parton distributions from selected data on the electromagnetic nucleon form factors. The extracted distributions can in particular be used to quantify the contribution to the proton spin from the total angular momentum carried by valence quarks, as well as their transverse spatial distribution inside the proton.

XXI International Workshop on Deep-Inelastic Scattering and Related Subjects-DIS2013, 22-26 April 2013

Marseilles, France

\footnotetext{
* Speaker.
} 


\section{Introduction}

Generalized parton distributions (GPDs) contain unique information about the structure of the proton. Most prominently, they quantify the transverse spatial distributions of partons in correlation with their longitudinal momentum [1,2]. Specific GPDs provide access to the angular momentum carried by partons, as becomes manifest from Ji's sum rule [3] and from spin-orbit correlations such as the change of the spatial parton distribution induced by transverse proton polarization [1].

Sum rules connect the GPDs for unpolarized quarks with the electromagnetic nucleon form factors according to

$$
F_{1}^{q}(t)=\int_{0}^{1} d x H_{v}^{q}(x, t), \quad F_{2}^{q}(t)=\int_{0}^{1} d x E_{v}^{q}(x, t),
$$

where we introduced the valence (quark minus antiquark) combinations

$$
H_{v}^{q}(x, t)=H^{q}(x, 0, t)+H^{q}(-x, 0, t), \quad E_{v}^{q}(x, t)=E^{q}(x, 0, t)+E^{q}(-x, 0, t)
$$

of the conventional GPDs $H^{q}(x, \xi, t)$ and $E^{q}(x, \xi, t)$ defined in [3]. The Dirac and Pauli form factors of the proton are given by charge weighted sums $F_{i}^{p}(t)=\sum_{q} e_{q} F_{i}^{q}(t)$ with $i=1,2$, and corresponding expressions for the neutron form factors are obtained using isospin symmetry. A Fourier transform to transverse position space turns $H_{v}^{q}$ into the probability density for finding a quark with flavor $q$, longitudinal momentum fraction $x$ and transverse position $\mathbf{b}$ in a proton, minus the corresponding probability density for antiquarks.

This contribution presents the work in ref. [4], where GPDs are determined from nucleon form factor data via the sum rules (1.1). In several ways, such a study is complementary to GPD extractions from hard exclusive processes like deeply virtual Compton scattering or meson production [5]. ( $i$ ) Form factor data extend to large values of $-t$, much beyond what can ever be reached in hard exclusive processes. (ii) The integrals in (1.1) are sensitive to a wide range of $x$, from about $10^{-3}$ to 0.6 and higher in our analysis. (iii) The sum rules (1.1) can be written in terms of GPDs at zero skewness $\xi$, which after Fourier transform admit a probability interpretation and obey much simpler positivity bounds than their counterparts with $\xi \neq 0$. (iv) On the downside, it is impossible to uniquely reconstruct the $x$ and $t$ dependence of GPDs from (1.1), which leaves us with an irreducible model dependence due to the functional forms assumed for $H_{v}^{q}(x, t)$ and $E_{v}^{q}(x, t)$.

\section{Form factor data}

For our analysis we have made a selection from the available data on electromagnetic form factors. The magnetic proton from factor $G_{M}^{p}(t)$ offers the largest reach in $t$, up to $-t \simeq 31 \mathrm{GeV}^{2}$. With experimental uncertainties of only a few $\%$ at small to intermediate $t$, quantitative control of two-photon exchange in the elastic ep cross section is essential for a reliable determination of this quantity. We use the data extracted in the global analysis of [6] and checked that it is compatible with the results of other recent work.

The scaled ratio $R^{p}=\mu_{p} G_{E}^{p} / G_{M}^{p}$ of electric and magnetic form factors can be extracted from elastic ep scattering data where either the polarization of the recoil proton is measured or the proton target is polarized. As is evident from figure 1, recoil polarization data from JLab Hall A published 

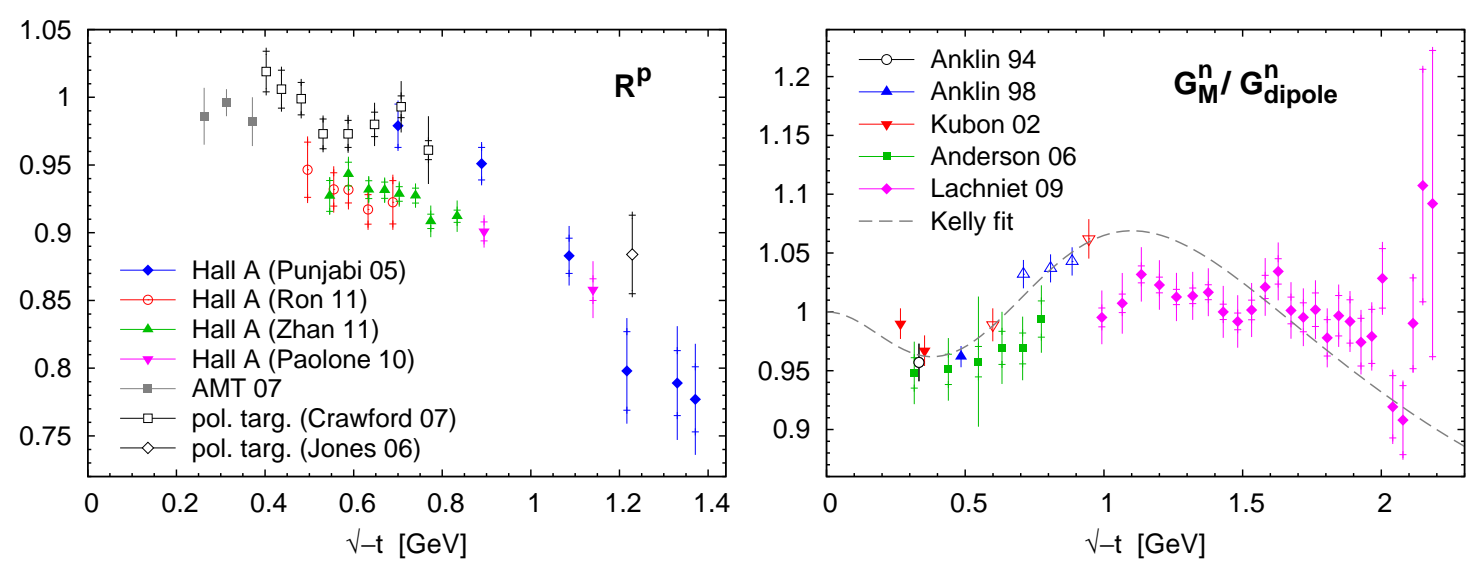

Figure 1: Left: Selection of recent data on the scaled from factor ratio $R^{p}=\mu_{p} G_{E}^{p} / G_{M}^{p}$. Right: Data on $G_{M}^{n}$, divided by the conventional dipole parameterization. Open symbols denote points that are omitted in our analysis (but clearly dominated a fit by Kelly in 2004, shown as a dashed line). References for the data sets are given in [4].

in 2010 and 2011 are clearly inconsistent with older data from the same hall and with measurements on polarized proton targets. We omit the polarized target data from our fit, while emphasizing that a clarification of the experimental situation is urgent.

Let us now turn to the neutron form factor data. For $G_{M}^{n}$ there is a number of data points from the 1990s that are inconsistent with more recent determinations (see figure 1) and have been excluded from our fits. For the electric neutron form factor $G_{E}^{n}$ or the ratio $R^{n}=\mu_{n} G_{E}^{n} / G_{M}^{n}$ there is an overall consistent data set, although only up to $-t \simeq 3.4 \mathrm{GeV}^{2}$. In our fits we also include the very precise determination of the squared charge radius $r_{n E}^{2}$ from neutron scattering on shell electrons in nuclear targets [7].

As a benchmark for our fit using GPDs, we have performed a simple global fit to the form factor data we have selected. We use the functional form

$$
F_{i}^{q}(t)=F_{i}^{q}(0)\left(1-a_{i q} t / p_{i q}\right)^{-p_{i q}}\left(1-b_{i q} t / q_{i q}\right)^{-q_{i q}}
$$

with $q=u, d$ and $i=1,2$, fixing 3 of the 16 parameters in this ansatz and fitting the remaining 13 . We obtain a global $\chi^{2}=122.3$ for 178 data points and a uniformly good description of the data for the proton and neutron form factors.

Furthermore, we have interpolated the data on the Sachs form factors and their ratios to a common set of $t$ values, where we can then compute mean values and errors for the form factors $F_{i}^{q}(t)$ and for any combination of them (see figure 2 below). Limited by the data on $R^{n}$ and $G_{E}^{n}$, this set covers a range up to $-t \simeq 3.4 \mathrm{GeV}^{2}$.

The electromagnetic form factors receive contributions also from strange quarks, i.e. from $F_{1}^{s}$ and $F_{2}^{s}$. We estimate these in a model that is consistent with determinations from lattice QCD and from parity violation in polarized elastic ep scattering (with data up to $-t \simeq 0.6 \mathrm{GeV}^{2}$ ). We find that the estimated size of $F_{1,2}^{s}(t)$ is comparable to the current uncertainties on $F_{1,2}^{u, d}(t)$, so that our poor knowledge of the strangeness sector does not yet have an impact on our determination of the GPDs for $u$ and $d$ quarks. 


\section{Fit of GPDs}

To extract valence quark GPDs from the form factor data we use an ansatz developed in our earlier work [8] (are related analysis can be found in [9]). We take an exponential form

$$
H_{v}^{q}(x, t)=q_{v}(x) \exp \left[t f_{q}(x)\right], \quad E_{v}^{q}(x, t)=e_{v}^{q}(x) \exp [\operatorname{tg}(x)],
$$

for the $t$ dependence, with $x$ dependent slopes

$$
\begin{aligned}
& f_{q}(x)=\alpha_{q}^{\prime}(1-x)^{3} \log (1 / x)+B_{q}(1-x)^{3}+A_{q} x(1-x)^{2}, \\
& g_{q}(x)=\alpha_{q}^{\prime}(1-x)^{3} \log (1 / x)+D_{q}(1-x)^{3}+C_{q} x(1-x)^{2} .
\end{aligned}
$$

A Fourier transform turns $H_{v}^{q}$ into a Gaussian in transverse space, where the average squared impact parameter $\left\langle\mathbf{b}^{2}\right\rangle_{x}^{q}=4 f_{q}(x)$ vanishes like $(1-x)^{2}$ for $x \rightarrow 1$ with our ansatz. This ensures that the distance $\mathbf{b} /(1-x)$ between the struck quark and the spectators remains on average finite in this limit, which is plausible for a system subject to confinement [1]. The $t=0$ limit of $H_{v}^{q}$ is given by the usual valence quark densities. Although these are relatively well known, there are important differences between different PDF sets at small and at large $x$. We take the ABM 11 NLO densities [10] at scale $\mu=2 \mathrm{GeV}$ as our default and repeat our analysis with a number of other recent parameterizations (CT 10, GJR 08, HERAPDF 1.5, MSTW 2008, NNPDF 2.2) as a cross check. We have verified that the CJ 12 parameterization, which was presented at this conference [11] and differs from other sets in the limiting behavior of $d_{v}$ at $x \rightarrow 1$, gives results within the range spanned by the other parameterizations in our fits. The $t=0$ limit of $E_{v}^{q}$ is unknown, and we make the ansatz

$$
e_{v}^{q}(x) \propto x^{-\alpha_{q}}(1-x)^{\beta_{q}}\left(1+\gamma_{q} \sqrt{x}\right),
$$

where the normalization is determined by the anomalous magnetic moments of the proton and neutron. The Fourier transform of $E_{v}^{q}$ describes the shift in the transverse spatial distribution of valence quarks that is induced by transverse proton polarization [1]. As a consequence, the size of $E_{v}^{q}$ relative to $H_{v}^{q}$ is limited by a positivity bound [12]. Imposing this bound in our fit significantly constrains the allowed parameter space, in particular by limiting the large- $x$ power $\beta_{q}$ in $e_{v}^{q}$ from below. Our best fit results are obtained by taking both $\beta_{u}$ and $\beta_{d}$ as small as possible, and we typically find $\beta_{u} \sim 4.5$ to 5 and $\beta_{d} \sim 5$ to 6 depending on the PDF set used for $H_{v}^{q}$. The precise data on $r_{n E}^{2}$ and $R^{n}$ favor a small flavor dependence with $\alpha_{u}^{\prime}>\alpha_{d}^{\prime}$ for the shrinkage parameter in $H_{v}^{q}$ and $E_{v}^{q}$. Our fitted values of $\alpha_{d}^{\prime}$ and $\alpha_{u}^{\prime}$ range from 0.68 to $1.0 \mathrm{GeV}^{-2}$, which is in line with expectations from Regge phenomenology, where the small- $x$ behavior of $H_{v}^{q}$ and $E_{v}^{q}$ is determined by the $\rho$ and $\omega$ trajectories.

We obtain a good overall fit of all form factor data, with a global $\chi^{2}=221.2$ for 178 data points if we use the ABM 11 PDFs. There are small discrepancies between fit and data for $G_{M}^{p}$ and $R^{p}$ below $-t=1 \mathrm{GeV}^{2}$, but only at the level of a few $\%$. In figure 2 we show the ratios $F_{1}^{d} / F_{1}^{u}$ and $F_{2}^{d} / F_{2}^{u}$, both normalized to 1 at $t=0$. As discussed in [8], our ansatz (3.1) and (3.2) reflects the Feynman mechanism by connecting the large- $t$ behavior of $F_{1}^{q}$ with the large- $x$ behavior of $q_{v}$. The stronger decrease of $F_{1}^{d}$ compared with $F_{1}^{u}$ seen in the data is thus naturally explained by the faster decrease of $d_{v}$ compared with $u_{v}$. Correspondingly, our fit predicts a faster decrease of $-F_{2}^{d}$ compared with $F_{2}^{u}$ for $\sqrt{-t}$ above $1.5 \mathrm{GeV}$. It will be interesting to see whether this is realized in Nature, once there are precise data for $G_{E}^{n}$ at higher $-t$. 

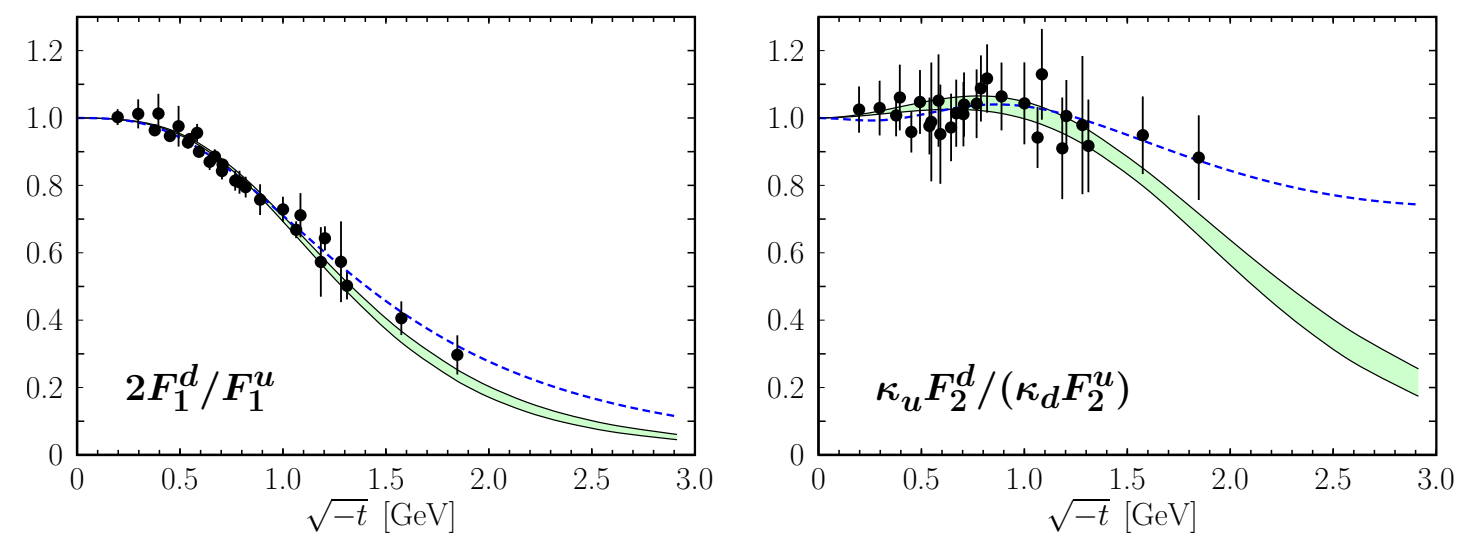

Figure 2: Normalized ratios of the $d$ and $u$ quark contributions to the Dirac and Pauli form factors of the proton. The band represents our default GPD fit and the dashed line the fit using (2.1). The data points have been obtained by interpolation of data on Sachs form factors and their ratios to a set of common $t$ values.

\section{Selected fit results}

Having extracted the GPDs $H_{v}^{q}$ and $E_{v}^{q}$, we can compute a variety of quantities of interest for the structure of the proton. The left panel in figure 3 shows the average of the distance between the struck parton and the spectators, defined by $d_{q}(x)=\left[\left\langle\mathbf{b}^{2}\right\rangle_{x}^{q}\right]^{1 / 2} /(1-x)$, and the right panel shows the average shift of this distance induced by transverse proton polarization, $s_{q}(x)=\left\langle\mathbf{b}^{y}\right\rangle_{x}^{q} /(1-x)$. A clear difference between valence $d$ and $u$ quarks is seen for both quantities.


Figure 3: Results of our default GPD fit for the transverse distribution of valence quarks in the proton. Left: average distance between the struck quark and the spectators. Right: average shift of this distance in the $y$ direction when the proton is polarized in the positive $x$ direction.

We can also evaluate the contribution of the total angular momentum carried by quarks minus the corresponding contribution from antiquarks. According to Ji's sum rule, this is given by

$$
J_{v}^{q}=\frac{1}{2} \int_{0}^{1} d x x\left[q_{v}(x)+e_{v}^{q}(x)\right]
$$


From our GPD fit we obtain

$$
J_{v}^{u}=0.230_{-0.024}^{+0.009}, \quad J_{v}^{d}=-0.004_{-0.016}^{+0.015}, \quad J_{v}^{u+d}=0.226_{-0.026}^{+0.004}, \quad J_{v}^{u-d}=0.233_{-0.038}^{+0.020}
$$

at the scale $\mu=2 \mathrm{GeV}$, where the errors include the parametric uncertainties of our default fit with the ABM 11 PDFs, as well as the variation of results if we employ different PDFs or change other details of the fit. ${ }^{1}$ These results are consistent with our previous work [8, 13], while using a much larger data set and giving a more comprehensive estimate of the uncertainty. They also agree with a recent determination [14] based on a phenomenological extraction of the Sivers distributions and a model for chromodynamic lensing, which gives $J_{v}^{u}=0.214_{-0.013}^{+0.009}$ and $J_{v}^{d}=-0.029_{-0.008}^{+0.021}$ if we add in quadrature the errors quoted in [14]. We find it encouraging that two model-dependent extractions using entirely different methods obtain consistent results. Subtracting from (4.2) the the helicity carried by valence quarks as obtained from the DSSV set of polarized parton densities [15], we obtain orbital angular momentum contributions

$$
L_{v}^{u}=-0.141_{-0.033}^{+0.025}, \quad L_{v}^{d}=0.114_{-0.035}^{+0.034}, \quad L_{v}^{u+d}=-0.027_{-0.039}^{+0.029}, \quad L_{v}^{u-d}=-0.255_{-0.061}^{+0.051}
$$

to the proton spin at $\mu=2 \mathrm{GeV}$, where we have added in quadrature the errors in (4.2) and those of the DSSV parameterization. In agreement with other analyses, we find a very small net orbital angular momentum, which results from a cancellation between significant individual contributions from $u$ and $d$ quarks. We emphasize that the results (4.2) and (4.3) are for the difference between quarks and antiquarks. Using electromagnetic form factors, we cannot access the contribution from antiquarks by itself. Evaluating the usual momentum sum rule for current PDF sets, we find that at $\mu=2 \mathrm{GeV}$ the longitudinal momentum carried by sea quarks in the proton is of similar size as the contribution from valence $d$ quarks. If this provides any guidance, then the contribution from sea quarks to Ji's sum rule may not be negligible.

\section{Conclusions}

The electromagnetic nucleon form factors are among the best known quantities describing proton structure. With the experimental precision that has been reached, several issues require further efforts before a consistent and reliable picture can be established. There are clear inconsistencies between results for the ratio $G_{E}^{p} / G_{M}^{p}$, whose resolution appears urgent to us. The quantitative control of two-photon exchange in the extraction of $G_{M}^{p}$, the measurement of $G_{E}^{n}$ or $G_{E}^{n} / G_{M}^{n}$ at higher $-t$, as well as a quantitative determination of the strangeness form factors, remain tasks for the future.

Using our selection of form factor data, we have determined a set of interpolated form factor values up to $-t \simeq 3.4 \mathrm{GeV}^{2}$, from which one can compute various form factor combinations of interest. We find that the power-law ansatz (2.1) gives an excellent and economical fit of all data. A good description of the data can be achieved with a fit of $u$ and $d$ valence quark GPDs, in which the positivity conditions relating $E$ and $H$ play an essential role. We have used the resulting GPDs to quantify the transverse distribution of valence quarks in the proton and to evaluate the total angular momentum carried by valence quarks, along with several other applications presented in [4].

\footnotetext{
${ }^{1}$ In [4] the upper error on $J_{v}^{d}$ is erroneously quoted to be +0.010 instead of +0.015 .
} 


\section{References}

[1] M. Burkardt, Impact parameter space interpretation for generalized parton distributions, Int. J. Mod. Phys. A 18 (2003) 173 [hep-ph/0207047].

[2] M. Diehl, Generalized parton distributions in impact parameter space, Eur. Phys. J. C 25 (2002) 223, Erratum-ibid. C 31 (2003) 277 [hep-ph/ 0205208 ].

[3] X.-D. Ji, Off forward parton distributions, J. Phys. G 24 (1998) 1181 [hep-ph/9807358].

[4] M. Diehl and P. Kroll, Nucleon form factors, generalized parton distributions and quark angular momentum, Eur. Phys. J. C 73 (2013) 2397 [arXiv: 1302 . 4604 ].

[5] F. Sabatié, From hard exclusive meson electroproduction to deeply virtual Compton scattering, these proceedings.

[6] J. Arrington, W. Melnitchouk and J. A. Tjon, Global analysis of proton elastic form factor data with two-photon exchange corrections, Phys. Rev. C 76 (2007) 035205 [arXiv: 0707.1861 ].

[7] J. Beringer et al. [Particle Data Group], Review of Particle Physics (RPP), Phys. Rev. D 86 (2012) 010001 .

[8] M. Diehl, T. Feldmann, R. Jakob and P. Kroll, Generalized parton distributions from nucleon form-factor data, Eur. Phys. J. C 39 (2005) 1 [hep-ph / 0408173 ].

[9] M. Guidal, M. V. Polyakov, A. V. Radyushkin and M. Vanderhaeghen, Nucleon form-factors from generalized parton distributions, Phys. Rev. D 72 (2005) 054013 [hep-ph/ 0410251 ].

[10] S. Alekhin, J. Blümlein and S. Moch, Parton Distribution Functions and Benchmark Cross Sections at NNLO, Phys. Rev. D 86 (2012) 054009 [arXiv: 1202. 2281].

[11] A. Accardi, The CJ12 parton distributions, these proceedings.

[12] M. Burkardt, Some inequalities for the generalized parton distribution E(x,0,t), Phys. Lett. B 582 (2004) 151 [hep-ph/0309116].

[13] M. Diehl, Generalized parton distributions: Recent results, AIP Conf. Proc. 842 (2006) 294 [hep-ph/0512201].

[14] A. Bacchetta and M. Radici, Constraining quark angular momentum through semi-inclusive measurements, Phys. Rev. Lett. 107 (2011) 212001 [arXiv: 1107.5755$].$

[15] D. de Florian, R. Sassot, M. Stratmann and W. Vogelsang, Extraction of spin-dependent parton densities and their uncertainties, Phys. Rev. D 80 (2009) 034030 [arXiv: 0904 . 3821]. 